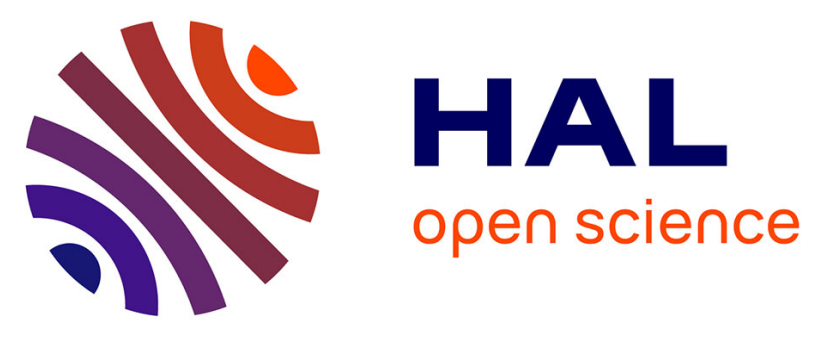

\title{
Bacteroides thetaiotaomicron uses a widespread extracellular DNase to promote bile-dependent biofilm formation
}

\author{
Nathalie Béchon, Jovana Mihajlovic, Anne-Aurélie Lopes, Sol \\ Vendrell-Fernández, Julien Deschamps, Romain Briandet, Odile Sismeiro, \\ Isabelle Martin-Verstraete, Bruno Dupuy, Jean-Marc Ghigo
}

\section{To cite this version:}

Nathalie Béchon, Jovana Mihajlovic, Anne-Aurélie Lopes, Sol Vendrell-Fernández, Julien Deschamps, et al.. Bacteroides thetaiotaomicron uses a widespread extracellular DNase to promote bile-dependent biofilm formation. Proceedings of the National Academy of Sciences of the United States of America, 2022, 119 (7), pp.e2111228119. 10.1073/pnas.2111228119 . hal-03571822

\section{HAL Id: hal-03571822 \\ https://hal.inrae.fr/hal-03571822}

Submitted on 15 Feb 2022

HAL is a multi-disciplinary open access archive for the deposit and dissemination of scientific research documents, whether they are published or not. The documents may come from teaching and research institutions in France or abroad, or from public or private research centers.
L'archive ouverte pluridisciplinaire HAL, est destinée au dépôt et à la diffusion de documents scientifiques de niveau recherche, publiés ou non, émanant des établissements d'enseignement et de recherche français ou étrangers, des laboratoires publics ou privés.

\section{(1) (1) $\$$}

Distributed under a Creative Commons Attribution - NonCommercial - NoDerivatives| 4.0 


\title{
Bacteroides thetaiotaomicron uses a widespread extracellular DNase to promote bile-dependent biofilm formation
}

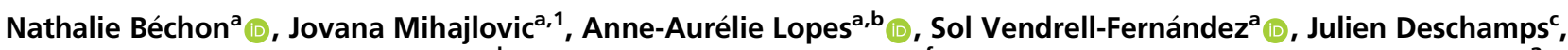

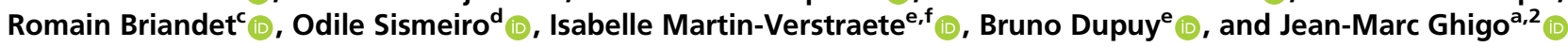

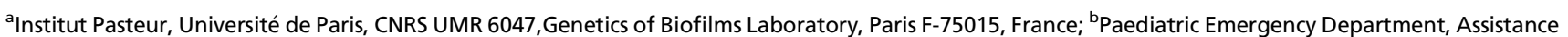 \\ Publique - Hôpitaux de Paris (AP-HP), University Hospital Robert Debré, Paris 75019, France; ' Université Paris-Saclay, Institut national de recherche pour \\ I'agriculture, I'alimentation et I'environnement (INRAE), AgroParisTech, Micalis Institute, Jouy-en-Josas 78350, France; 'Institut Pasteur, Université de Paris, \\ Transcriptome and Epigenome Platform, Biomics, Paris F-75015, France; 'Institut Pasteur, Université de Paris, CNRS UMR 6047, Pathogenesis of Bacterial \\ Anaerobes Laboratory, Paris F-75015, France; and ${ }^{f}$ Institut Universitaire de France, Paris 75006, France
}

Edited by Eric Martens, Microbiology and Immunology, University of Michigan, Ann Arbor, Ml; received June 17, 2021; accepted December 17, 2021 by Editorial Board Member Thomas J. Silhavy

\begin{abstract}
Bacteroides thetaiotaomicron is a gut symbiont that inhabits the mucus layer and adheres to and metabolizes food particles, contributing to gut physiology and maturation. Although adhesion and biofilm formation could be key features for $B$. thetaiotaomicron stress resistance and gut colonization, little is known about the determinants of $B$. thetaiotaomicron biofilm formation. We previously showed that the $B$. thetaiotaomicron reference strain VPI-5482 is a poor in vitro biofilm former. Here, we demonstrated that bile, a gut-relevant environmental cue, triggers the formation of biofilm in many $B$. thetaiotaomicron isolates and common gut Bacteroidales species. We determined that bile-dependent biofilm formation involves the production of the DNase BT3563 or its homologs, degrading extracellular DNA (eDNA) in several $B$. thetaiotaomicron strains. Our study therefore shows that, although biofilm matrix eDNA provides a biofilm-promoting scaffold in many studied Firmicutes and Proteobacteria, BT3563-mediated eDNA degradation is required to form $B$. thetaiotaomicron biofilm in the presence of bile.
\end{abstract}

biofilm | Bacteroides thetaiotaomicron | DNase | gut microbiota

B acteroides thetaiotaomicron, one of the most abundant bacterial gut symbionts, is involved in the degradation of complex polysaccharides and the maturation of the host immune system (1-3). B. thetaiotaomicron colonizes the gel-like mucus layer of intestinal epithelia cells (IECs) in both healthy and diseased conditions, and also adheres to food particles (4-6), suggesting that adhesion to host surfaces and the formation of bacterial biofilm aggregates could play an important biological role. Consistently, the transcriptional profile of $B$. thetaiotaomicron colonizing the mouse gut is more similar to $B$. thetaiotaomicron in vitro biofilm than to in vitro planktonic culture (7). Whereas the reference strain VPI-5482 only forms poor biofilms in vitro, several mutations leading to increased aggregation and biofilm capacity in vitro were recently identified, revealing the importance of capsular polysaccharides and type $\mathrm{V}$ pilus in mediating adhesion $(8,9)$. Moreover, polysaccharide utilization loci (PUL) have been proposed to mediate Bacteroides adhesion to substrates. PULs are surface structures involved in sugar degradation that are composed of at least one TonB-dependent receptor and one cell surface lipoprotein binding a glycan substrate $(3,10)$. Mucin-O-glycan-degrading PUL might mediate adhesion to mucin and therefore favor mucus colonization in the gut upon bile stimulation $(3,7)$. Although several gut environmental cues and compounds, including antibiotics, oxygen, metal concentration, mucins, immunoglobulin A, and bile (11-17), were shown to modulate bacterial biofilm capacity in various bacterial species, gut-relevant conditions promoting $B$. thetaiotaomicron biofilm formation are still poorly understood.
In the closely related Bacteroides fragilis species, both subinhibitory concentrations of the antibiotic enrofloxacin and bile were independently shown to increase in vitro biofilm formation, with consequences on $B$. fragilis physiology $(15,18)$. In this study, we showed that exposure to subinhibitory concentrations of bile extract induced biofilm formation in $B$. thetaiotaomicron strain VPI-5482 and in the majority of tested B. thetaiotaomicron clinical isolates and several common gut Bacteroidales species. Using transposon mutagenesis, we identified a $B$. thetaiotaomicron VPI5482 operon, BT3560-3563, involved in bile-dependent biofilm formation. We showed that BT3563 is an extracellular DNase that is well-represented in B. thetaiotaomicron species and that it degrades extracellular DNA (eDNA) in biofilms formed in the presence of bile. Our study therefore shows that, in contrast to

\section{Significance}

Biofilms are communities of surface-attached bacteria exhibiting biofilm-specific properties. Although anaerobic biofilms impact health, industry, and environment, they are mostly studied in aerobic bacterial species. Here, we studied biofilm formation in Bacteroides thetaiotaomicron, an anaerobic gut symbiont degrading diet sugars and contributing to gut maturation. Although $B$. thetaiotaomicron adhesion contributes to intestinal colonization, little is known about the determinants of its biofilm capacities. We identified that bile is a physiologically relevant gut signal inducing biofilm formation in B. thetaiotaomicron and other gut Bacteroidales. Moreover, we showed that, in contrast to the known scaffolding role of extracellular DNA, bile-dependent biofilm requires a DNase degrading matrix DNA, thus revealing a previously unrecognized factor contributing to the adhesion capacity of major gut symbionts.

Author contributions: N.B., J.M., and J.-M.G. designed research; N.B., J.M., A.-A.L., S.V.-F., J.D., and O.S. performed research; I.M.-V. and B.D. contributed new reagents/ analytic tools; N.B., J.M., A.-A.L., J.D., R.B., O.S., and J.-M.G. analyzed data; I.M.-V. and B.D. contributed to the initial experiments and provided advice; and N.B., J.M., and J.-M.G. wrote the paper.

The authors declare no competing interest.

This article is a PNAS Direct Submission. E.M. is a guest editor invited by the Editorial Board.

This article is distributed under Creative Commons Attribution-NonCommercialNoDerivatives License 4.0 (CC BY-NC-ND).

${ }^{1}$ Present address: Center for Microbiology and Environmental Systems Science, Department of Microbiology and Ecosystem Science, Division of Microbial Ecology, University of Vienna, 1010 Vienna, Austria.

${ }^{2}$ To whom correspondence may be addressed. Email: jmghigo@pasteur.fr.

This article contains supporting information online at http://www.pnas.org/lookup/ suppl/doi:10.1073/pnas.2111228119/-/DCSupplemental.

Published February 10, 2022. 
the biofilm-promoting role of bacterial extracellular DNA in many species (19), degradation of eDNA by BT3563 or by other extracellular nucleases may be a widespread requirement to achieve biofilm formation in the presence of bile, as shown with B. thetaiotaomicron.

\section{Results}

Bile Induces Biofilm Formation in B. thetaiotaomicron and Several Bacteroidales. To identify gut-relevant conditions inducing biofilm formation in B. thetaiotaomicron VPI-5482, we supplemented brain heart infusion supplemented (BHIS) growth medium with various components of the normal intestinal environment, including sugars $(0.5 \%$ D-glucose, D-mannose, D-rhamnose, D-cellobiose, and D-maltose), hemin $(25 \mathrm{mg} / \mathrm{L}$ and $50 \mathrm{mg} / \mathrm{L})$, mucin $(0.1 \%$ and $0.5 \%)$, or a mix of bovineovine bile extract $(0.1 \%, 0.5 \%$, and $1 \%)$ (hereafter referred to as bile) for $24 \mathrm{~h}$ (SI Appendix, Fig. S1). We showed that addition of bile increased biofilm formation at $24 \mathrm{~h}$ without impacting viable cell count (minimal inhibitory concentration for bile is 4\%) (SI Appendix, Figs. S1 and S2). This increase was maximal at $48 \mathrm{~h}$ in the presence of 0.3 to $1 \%$ bile (Fig. $1 A$ ). We then tested biofilm formation at $48 \mathrm{~h}$ in the presence of $0.5 \%$ bile in a collection of $B$. thetaiotaomicron clinical isolates (8) (SI Appendix, Table S1) and a representative strain of the most common gut Bacteroides and Parabacteroides species (Fig. $1 B$ and $C$ ). We showed that, except for $B$. thetaiotaomicron strain jmh71 and the tested Bacteroides vulgatus strain, all strains displayed an increased biofilm phenotype (Fig. $1 B$ and $C$ ), indicating that bile is a widespread inducer of biofilm formation among gut Bacteroidales species.
Exposure to Bile Induced a Major Transcriptional Shift but Did Not Reveal Biofilm Functions. To understand the impact of bile on B. thetaiotaomicron physiology, we performed a RNA-sequencing (RNA-seq) analysis comparing $B$. thetaiotaomicron grown overnight in the presence or absence of $0.5 \%$ bile. We found that bile induces a widespread transcriptional shift, with $17 \%$ genes showing a significant change (Dataset $\mathrm{S} 1 A$ ). In the presence of bile, out of 4,816 genes, 378 and 429 genes were significantly up- and down-regulated, respectively (Dataset $\mathrm{S} 1 B$ and $C$ ). About half of these genes could be assigned a functional Clusters of Orthologous Groups (COG) category, spanning almost all COG categories (Dataset $\mathrm{S} 1 D$ ). The 5 genes showing the highest fold change (>50) are organized in two operons, BT2793-2795, encoding a bile-specific tripartite multidrug efflux system , and BT0691-0692, predicted to encode an outer membrane protein and a calcineurin superfamily phosphohydrolase, respectively. Consistently, both of these loci were previously shown to be important for fitness under bile salt stress (20) and are probably involved in bile tolerance. Although we did not observe any induction of genes encoding capsular polysaccharides $(21,22)$ or type $\mathrm{V}$ pilin homo$\operatorname{logs}(23)$, that were previously shown to contribute to $B$. thetaiotaomicron biofilm formation $(8,9)$, several PUL involved in mucin-O-glycan and host-derived sugar degradation (3) that could contribute to adhesion to the mucus layer were up-regulated, suggesting that bile exposure might increase $B$. thetaiotaomicron adhesion capacity also in vivo. These results showed that exposure to bile leads to important physiological adaptations in $B$. thetaiotaomicron that might increase its colonization capacity. This transcriptomic approach did not, however, reveal any regulation of genes encoding known B. thetaiotaomicron determinants of biofilm formation in vitro.

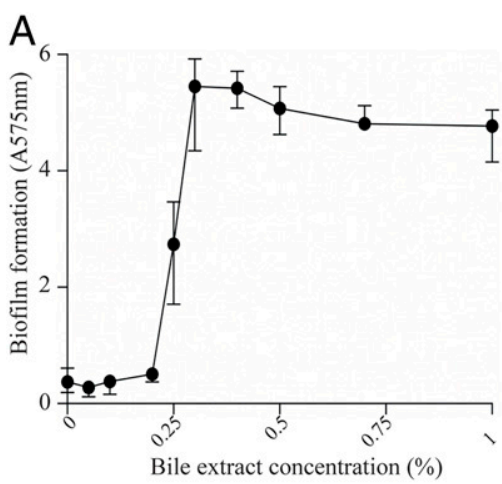

B

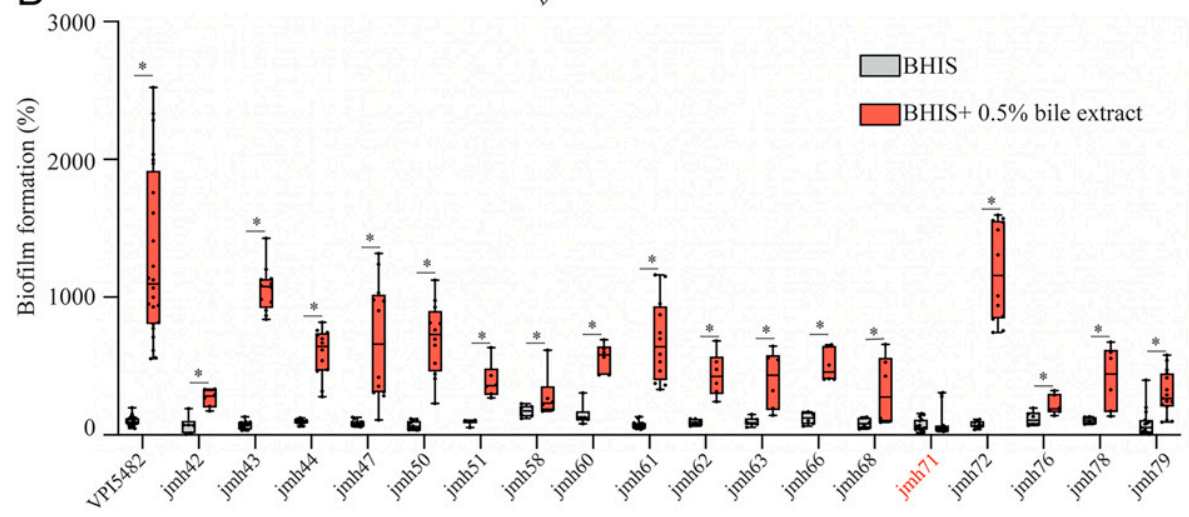

Fig. 1. Bile extract promotes biofilm formation of several Bacteroidales isolates. (A) B. thetaiotaomicron VPI-5482 48-h biofilm formation as a function of bile extract concentration. Mean of six biological replicates, error bars represent SD. (B and $C$ ) A 96-well plate crystal violet 48-h biofilm assay. Mean of $B$. thetaiotaomicron VPI-5482 grown in BHIS is adjusted to $100 \%$. Minimum-maximum (min-max) boxplot of 6 to 12 biological replicates for each strain. ${ }^{*} P$ value $<0.05, * * P$ value $<0.005$, Mann-Whitney $U$ test. 


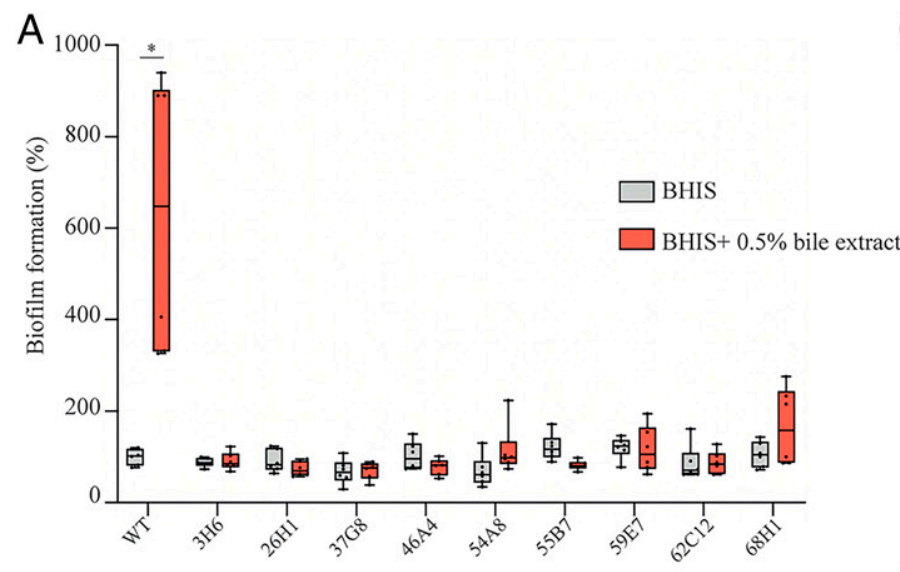

C
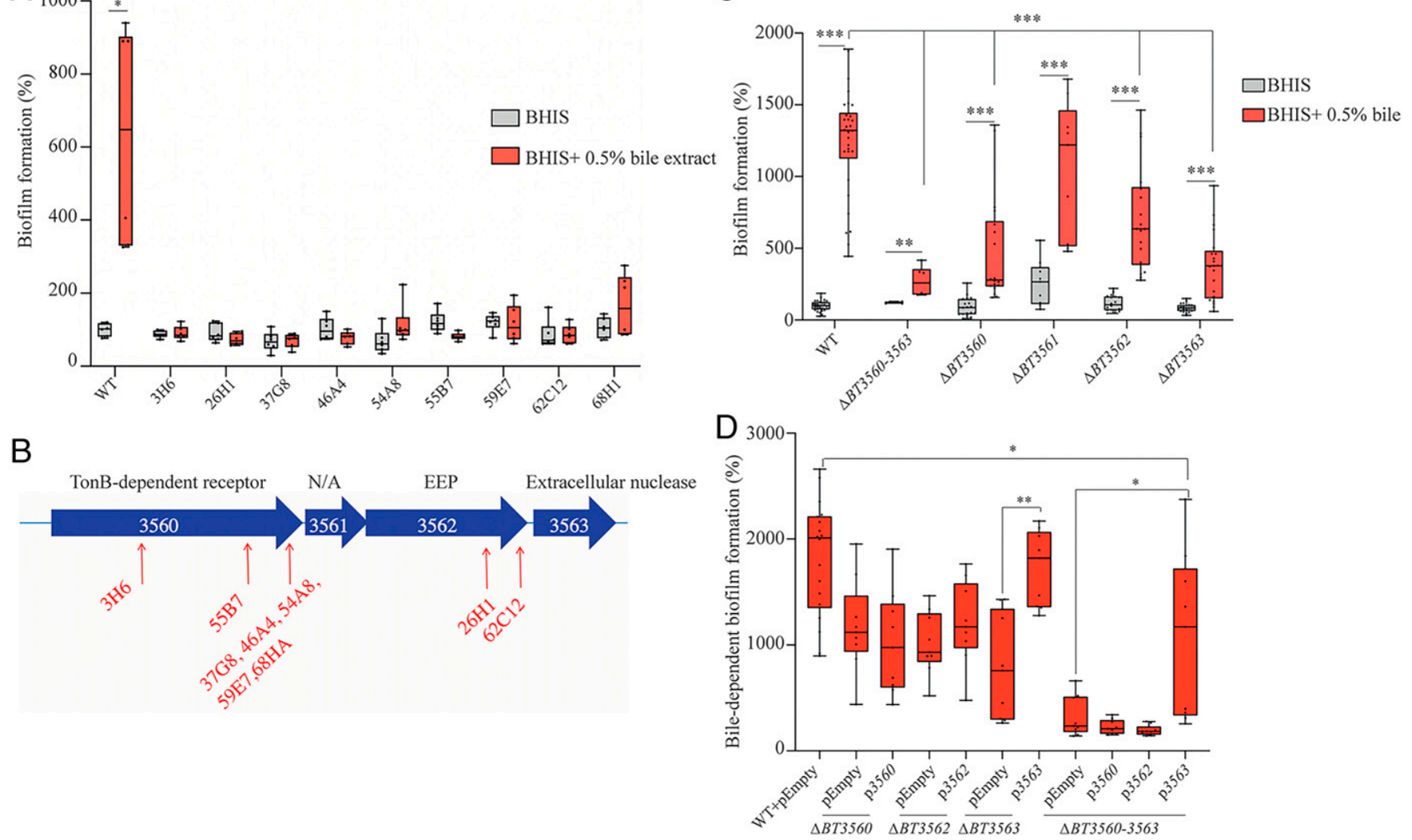

Fig. 2. $B T 3560-3563$ is required for bile-dependent biofilm formation. ( $A$ and $C$ ) A 96-well plate crystal violet biofilm assay after 48 -h growth in BHIS or BHIS $+0.5 \%$ bile extract. $(B)$ Schema of the BT3560-3563 genetic locus. Red arrows indicate transposon insertion points. N/A, not annotated; EEP, exonuclease/endonuclease/phosphatase family. (D) A 96-well plate crystal violet biofilm assay after 48 -h growth in BHIS + 0.5\% bile extract. WT in BHIS is not represented. $(A, C$, and $D)$ Mean of WT in BHIS is adjusted to $100 \%$. Min-max boxplot of 6 to 18 biological replicates for each strain, each replicate is the mean of two technical replicates. $* P$ value $<0.05, * * P$ value $<0.005, * * * P$ value $<0.0005$, Mann-Whitney $U$ test.

Identification of an Operon Involved in Bile-Dependent Biofilm Formation. To identify the genetic determinants involved in bile-dependent biofilm formation, we performed a random transposon mutagenesis in $B$. thetaiotaomicron VPI-5482 and screened for mutants failing to produce biofilm in the presence of $0.5 \%$ bile. We identified 9 biofilm-negative transposon mutants out of 6,510 mutants screened (Fig. $2 A$ ), all corresponding to transposon insertions in a potential operon ( $S I$ Appendix, Fig. S3) encoding a TonB-dependent receptor (BT3560, 7 insertions), a hypothetical protein (BT3561), and two putative membrane-associated lipoproteins with nuclease activity (BT3562, 2 insertions and BT3563) (Fig. 2B). Whereas deletion of $B T 3561$ had no impact on biofilm formation, the deletion of BT3560, BT3562, BT3563, or of the whole BT35603563 region significantly reduced bile-dependent biofilm formation compared to WT without an associated growth defect (Fig. $2 C$ and SI Appendix, Fig. S4). Since crystal violet stains both cells and extracellular matrix (ECM), we resuspended biofilms and numbered colony forming units (CFUs) and showed that $\triangle B T 3560-3563$ reduction in biofilm formation was due to a reduction of cell number and not ECM production (SI Appendix, Fig. S5). Complementation of $\triangle B T 3563$ and $\triangle B T 3560-3563$ mutants by $\mathrm{p} B T 3563$, a plasmid constitutively expressing $B T 3563$, restored bile-dependent biofilm formation (Fig. 2D), whereas $\triangle B T 3560$ or $\triangle B T 3562$ strains were not complemented upon addition of $\mathrm{p} B T 3560$ or $\mathrm{p} B$ T3562, suggesting that deletion of $B T 3560$ and BT3562 might have a polar effect on the downstream BT3563 gene (Fig. 2D). However, since a $\triangle B T 3560-3563$ mutant formed slightly but reproducibly less biofilm than a $\triangle B T 3563$ simple mutant, BT3560 and BT3562 might also contribute to biofilm formation in the presence of bile. Taken together, these results showed that BT3563 is necessary for proper bile-dependent biofilm formation of B. thetaiotaomicron VPI-5482.

BT3563 is an Extracellular DNase Degrading eDNA in Biofilms Formed in the Presence of Bile. To investigate the potential nuclease activity of BT3563, we exposed genomic DNA to $B$. thetaiotaomicron 24-h and 48-h bile-free culture supernatant. We showed that DNA was degraded after an overnight incubation with a wild-type (WT) supernatant. This degradation was abolished when we used a $\Delta B T 3563$ strain carrying the empty vector (Fig. $3 A$ and SI Appendix, Fig. S6). Complementation with pBT3563 plasmid, constitutively expressing BT3563, restored DNA degradation, confirming the DNase activity of BT3563 (Fig. $3 A$ ). To confirm the contribution of BT3563 extracellular DNase to bile-dependent biofilm formation, we complemented the absence of BT3563 in $\triangle B T 3560-3563$ and $\triangle B T 3563$ strains by adding purified DNase I to the growth medium. We observed an increase of bile-dependent biofilm formation in $\triangle B T 3560$ 3563 and $\triangle B T 3563$ strains in the presence of DNase I, but a decrease in WT biofilm formation (Fig. $3 B$ ). This prompted us to measure the concentration of eDNA in the ECM of B. thetaiotaomicron biofilms formed in the presence of bile. We showed that, whereas deletion of BT3563 did not significantly impact ECM protein concentration (SI Appendix, Fig. S7), it increased eDNA concentration compared to WT. Upon addition of $\mathrm{p} B T 3563$ in trans, the eDNA concentration was reduced back to WT level, which is consistent with BT3563-dependent degradation of DNA in the ECM (Fig. 3C). Addition of bile was not necessary to 

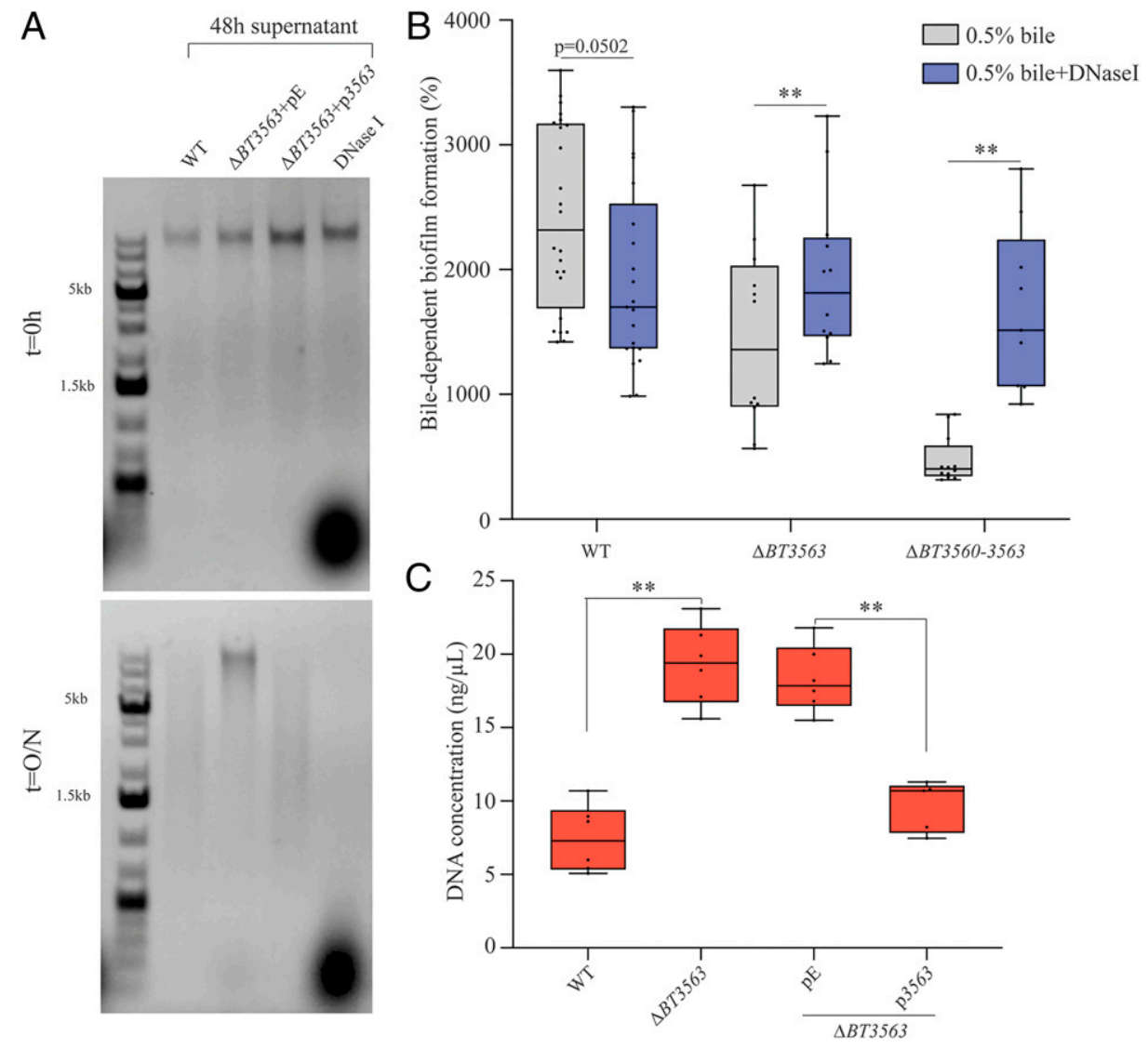

Fig. 3. BT3563 degrades nucleic acids in the extracellular matrix of bile-dependent biofilms. (A) Incubation of $B$. thetaiotaomicron genomic DNA ( 23 ng/ $\mu \mathrm{L}$ final concentration) with the supernatant of 48-h bile-free cultures, as indicated, and loaded immediately on a $1 \%$ agarose gel $(\mathrm{t}=0 \mathrm{~h})$ and after an overnight incubation at $37^{\circ} \mathrm{C}(\mathrm{t}=\mathrm{O} / \mathrm{N})$. A control using DNase I rather than supernatant is shown in the last lane. $\mathrm{pE}$, empty vector; p3563, vector constitutively expressing BT3563. (B) A 96-well plate crystal violet biofilm assay after 48-h growth in BHIS $+0.5 \%$ bile extract, with or without DNase I. Mean of WT in BHIS is adjusted to $100 \%$ (not represented). Min-max boxplot of 9 to 21 biological replicates for each strain, each replicate is the mean of two technical replicates. $* * P$ value $<0.005$, Wilcoxon matched-pairs signed rank test. (C) Extracellular DNA concentration (ng/ $\mu \mathrm{L}$ ) in the purified extracellular matrix of bile-dependent biofilms grown in BHIS $+0.5 \%$ bile extract for $48 \mathrm{~h}$. Min-max boxplot of 6 biological replicates for each strain. ${ }^{* * P}$ value $<0.005$, Mann-Whitney $U$ test. $\mathrm{pE}$, empty vector; $\mathrm{p} 3563$, vector constitutively expressing $B T 3563$.

observe BT3563-mediated DNA degradation (Fig. 3A), and our transcriptomic analysis showed no increase of $B T 3563$ transcription in the presence of bile (Dataset $\mathrm{S} 1 A$ ), suggesting that bile might not directly impact BT3563 production or activity. We then assessed the impact of bile on eDNA concentration itself and observed an increase of eDNA concentration in B. thetaiotaomicron supernatant of cultures grown in the presence of bile, indicating that bile could increase eDNA release (SI Appendix, Fig. S8). These results showed that BT3563 is an extracellular DNase that degrades eDNA in the biofilm matrix and that bile exposure increases the release of eDNA, which might be one of the mechanisms by which bile exposure favors biofilm formation.

Extracellular DNase Activity Is Necessary to Observe Maximum BileDependent Biofilm Formation. To analyze the distribution and abundance of eDNA in the ECM of $B$. thetaiotaomicron biledependent biofilms, we labeled eDNA with TOTO-1 dye and imaged 48-h 96-well plate biofilms by confocal laser scanning microscopy (CLSM). For each condition, a representative well is shown in Fig. 4; the five others are shown in SI Appendix, Fig. S9; a three-dimensional (3D) reconstruction of the biofilm for one well is shown in SI Appendix, Fig. S10 and quantification of TOTO-1 and SYTO61 fluorescence is shown in $S I$ Appendix, Fig. S11. In the presence of bile, B. thetaiotaomicron biofilm was expectedly denser and more structured than in the absence of bile (Fig. $4 A$ and $B$ and SI Appendix, Figs. S9 and
S10). Extracellular nucleic acids were 20 times more abundant in the presence of bile, when correcting for cell abundance (SI Appendix, Fig. S11C) and they were distributed diffusely in the entire biofilm, with spots potentially corresponding to dead cells (Fig. $4 A$ and $B$ and SI Appendix, Figs. S9 and S10). To determine the nature of these nucleic acids, we grew $B$. thetaiotaomicron biofilms in the presence of bile and DNase I or RNase I. Whereas RNase I treatment had little impact on $B$. thetaiotaomicron biofilms (Fig. $4 C$ and SI Appendix, Figs. S9-S11), addition of DNase I decreased by 20-fold the amount of extracellular nucleic acids detected (Fig. $4 D$ and $S I$ Appendix, Fig. S11C), close to the levels observed for biofilms formed in the absence of bile. This confirms that DNA was the most abundant nucleic acid in the ECM upon bile induction. Addition of DNase I also led to a loss of B. thetaiotaomicron biofilm 3D structure, suggesting eDNA might be an important structuring component of the ECM (Fig. $4 D$ and SI Appendix, Figs. S9 and S10). We then showed that, whereas the $\triangle B T 3563$ mutant formed scattered biofilms in the presence of bile, with dispersed or small clumps of cells (Fig. $4 E$ and $F$ and $S I$ Appendix, Figs. S9 and S10), addition of DNase I during growth of $\triangle B T 3563$ restored a WT homogenous and dense biofilm phenotype (Fig. $4 D$ and $G$ and SI Appendix, Figs. S9 and S10). One hypothesis would be that DNase activity might be required to prevent repulsion between clumps of cells, or between cells and some components of the extracellular matrix, allowing the 


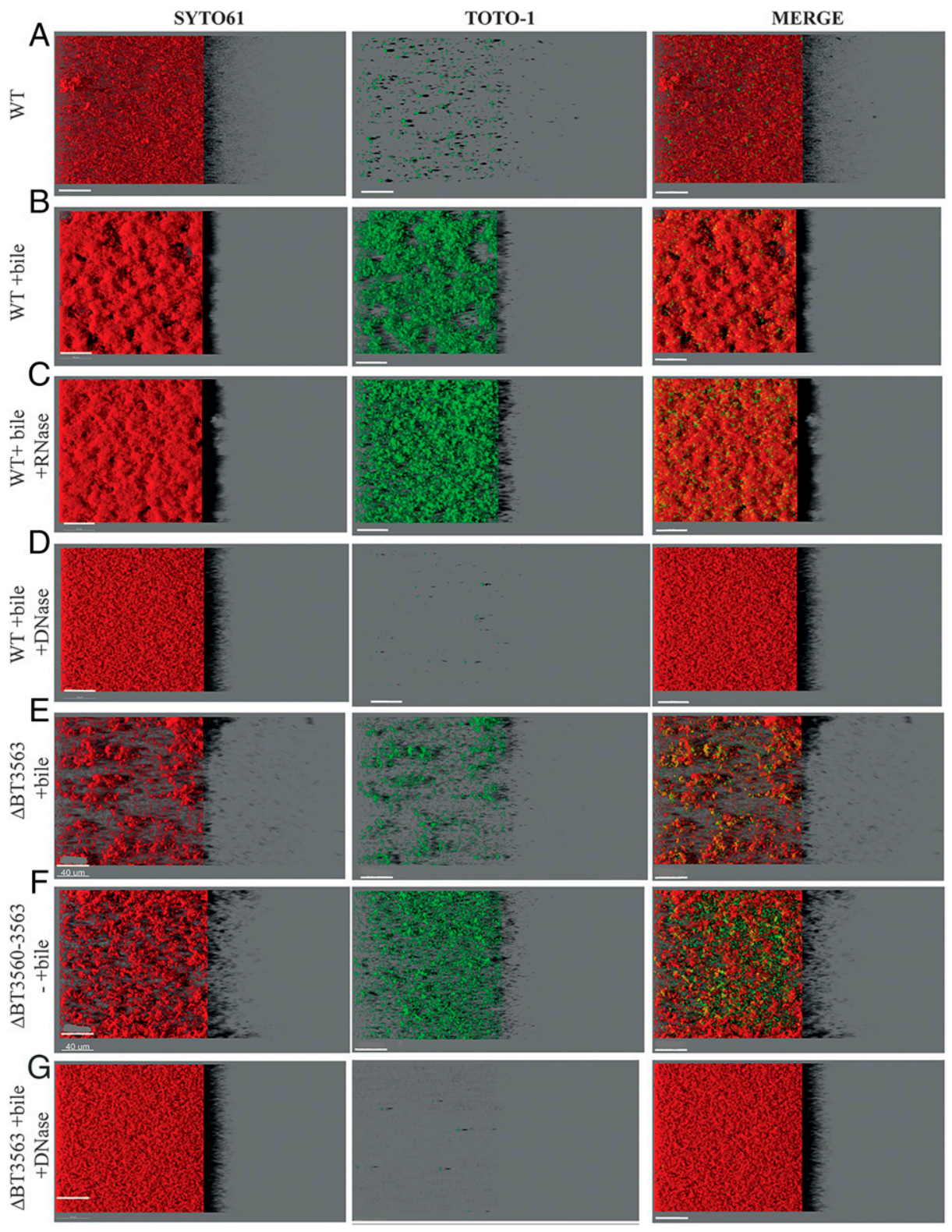

Fig. 4. ( $A-G)$ Visualization of $B$. thetaiotaomicron biofilms. IMARIS easy $3 D$ projections from CLSM images of $B$. thetaiotaomicron biofilms grown in plates in the absence or presence of $0.5 \%$ bile extract ("+bile"), RNase I ("+RNase"), or DNase I ("DNase"). Cells are labeled with SYTO61 dye (Left, in red). eDNA and dead cells are labeled with TОтО-1 (Middle, in green). The merged image is shown on the Right. For each image, the virtual shadow projection of the biofilm is shown on the Right. (Scale bars, $40 \mu \mathrm{m}$.)

formation of denser structures. By contrast to eDNA quantification in the extracted ECM, we saw no increase in TOTO-1 labeling of $\triangle B T 3563$ or $\triangle B T 3560-3563$ mutants compared to WT in the presence of bile (SI Appendix, Fig. S11C), which could be due to a lack of sensitivity of eDNA quantification by CLSM. These results demonstrated that DNA, rather than RNA, contributes to biofilm structure in the presence of bile and that the presence of either BT3563 or the addition in culture of DNase I lead to the formation of denser bacterial structures, allowing maximum biofilm formation.

BT3563 Homologs Are Involved in Bile-Dependent Biofilm Formation of $\boldsymbol{B}$. thetaiotaomicron Clinical Isolates. To further investigate the correlation between BT3563 and bile-dependent biofilm, we sequenced the whole genome of the $18 \mathrm{~B}$. thetaiotaomicron jmh isolates (24). We found a BT3563 homolog in all tested strains, even in $B$. thetaiotaomicron jmh71, the only strain that did not form biofilms in the presence of bile (SI Appendix, Table S2), suggesting jmh71 could be lacking additional factors needed for bile-dependent biofilm formation apart from BT3563. In 11 out of the 18 jmh strains (jmh42, 44, 47, 50, 51, 58, 61, 66, 76, 78, and 79), the homology with VPI-5482 BT3563 exceeded $80 \%$ homology (SI Appendix, Table S2) and the genomic region around the detected BT3563 homolog gene was similar to that of VPI-5482 and included homologs of genes BT3558 to $B T 3564$ (as depicted in Fig. 5A). In the 7 other strains (jmh43, $60,62,63,68,71$, and 72), the homology with VPI-5482 BT3563 was weaker (34\% identity, SI Appendix, Table S2), and the genetic environment of their BT3563 homologs differed, including a homolog of BT3559, BT3560, BT3564, as well as a gene coding for a protein carrying a Bacteroides-associated carbohydrate binding often $\mathrm{N}$-terminal (BACON) domain Fig. 5A. We also showed that Bacteroides ovatus and Bacteroides uniformis are the only Bacteroidales strains tested with a 

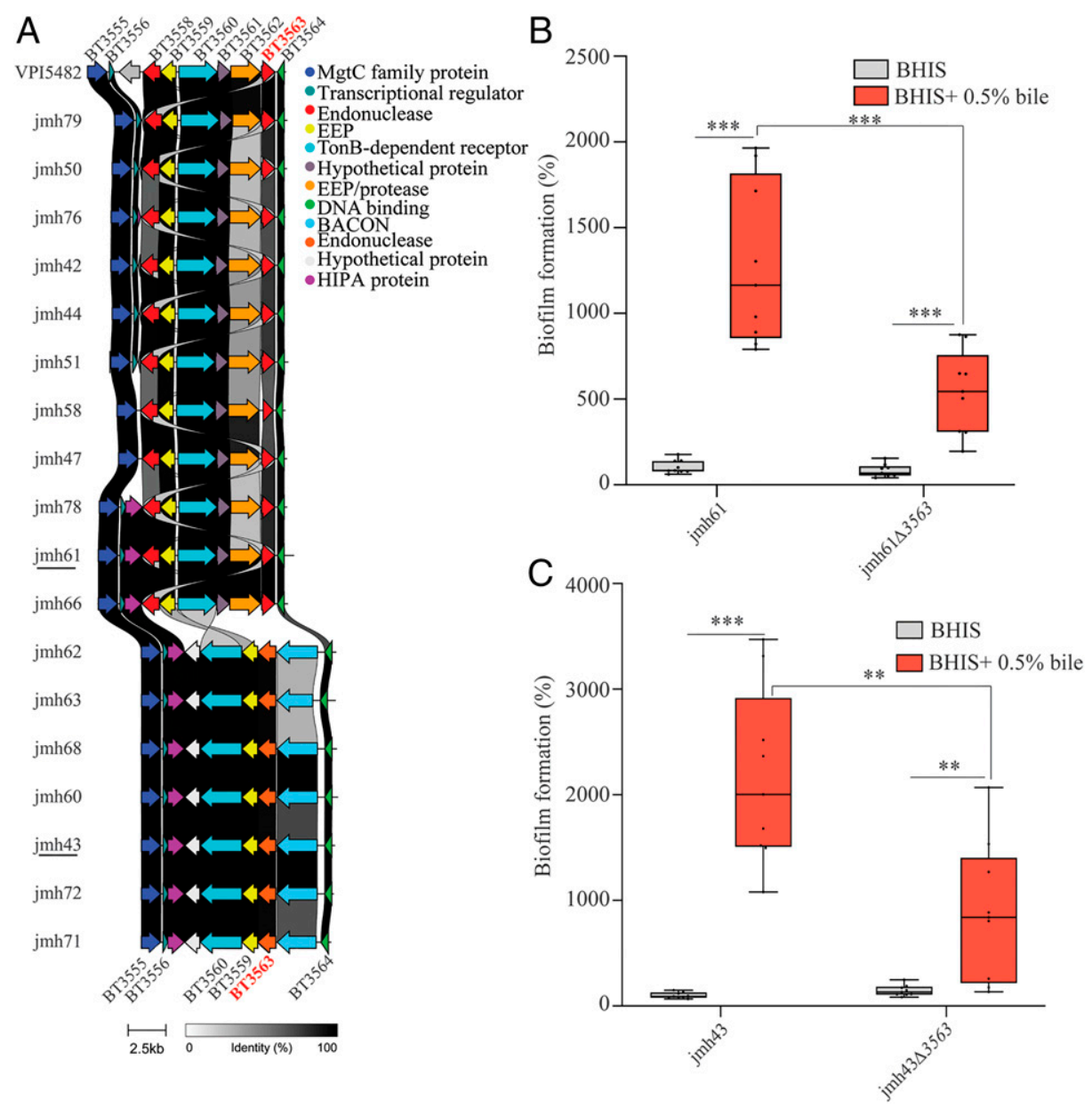

Fig. 5. $B T 3563$ homologs are involved in bile-dependent biofilm formation of $B$. thetaiotaomicron clinical isolates. ( $A$ ) Comparison of the genetic organization around BT3563 homologs in B. thetaiotaomicron isolates. EEP, endonuclease/exonuclease/phosphatase superfamily; BACON, Bacteroides-associated carbohydrate binding often N-terminal; HIPA, serine/threonine-protein kinase toxin HipA. BT3563 homologs sharing more than $80 \%$ sequence identity with VPI-5482 BT3563 are shown in red (for jmh42, 44, 47, 50, 51, 58, 61, 66, 76, 78, and 79) and BT3563 homologs sharing $34 \%$ sequence identity with VPI-5482 BT3563 are shown in orange (for jmh43,60,62,63, 68, 71, and 72). ( $B$ and $C$ ) A 96-well plate crystal violet biofilm assay after 48-h growth in BHIS or BHIS + $0.5 \%$ bile extract. Mean of jmh61 or jmh43 in BHIS is adjusted to $100 \%$. Min-max boxplot of nine biological replicates for each strain; each replicate is the mean of two technical replicates. ${ }^{*} P$ value $<0.005, * * * P$ value $<0.0005$, Mann-Whitney $U$ test.

BT3555-BT3564 region similar to $B$. thetaiotaomicron VPI5482, including a homolog of BT3560, BT3562, and BT3563 (SI Appendix, Fig. S12). In the other strains tested, this region is either completely missing (Parabacteroides distasonis) or present without a BT3563 homolog (B. fragilis, B. vulgatus, Bacteroides eggerthii) (SI Appendix, Fig. S12). A more comprehensive search revealed that BT3563 homologs were present in many diverse Bacteroidales, and at least in one example of Flavobacterium columnare and Salinivirga cyanobacteriivorans, non-Bacteroidales Bacteroidetes. However, the synteny of the BT3560-3564 region was conserved only in strains from the Bacteroides, Prevotella, Alloprevotella, Porphyromonas, and Alistipes genera (SI Appendix, Fig. S13). We tested the contribution of BT3563 homologs to B. thetaiotaomicron bile-dependent biofilm formation in two randomly selected strains, jmh61 and jmh43. In these strains, the BT3563 proteins share, respectively, $87 \%$ and $34 \%$ of protein identity with VPI-5482 BT3563, and a different genetic organization around the BT3563 homolog is observed (SI Appendix, Table S2 and Fig. 5A). We showed that deletion of the BT3563 homolog in both jmh61 and jmh43 led to a reduction of bile-dependent biofilm formation (Fig. $5 \mathrm{~B}$ and $C$ ), indicating that the contribution of VPI-5482 BT3563 extracellular DNase to bile-dependent biofilm formation is a widespread property of $B$. thetaiotaomicron species.

\section{Discussion}

In this report, we showed that physiological concentrations of bile extract induce the formation of biofilm in almost all tested B. thetaiotaomicron strains, as well as in representative Bacteroidales species. This widespread induction by a compound exclusive to the intestinal environment suggests that biofilm formation might be an important aspect of Bacteroidales biology in the gut. Bile concentration in the gut can range between 0.2 and $2 \%(25)$, which is consistent with the concentrations required to induce maximum $B$. thetaiotaomicron biofilm formation $(0.3$ to $1 \%)$. However, the concentration of intestinal bile is highly variable, depending on the individual, time of the day, diet, and the precise localization within the gut. Moreover, bile composition changes along the intestine and also depends on environmental parameters such as diet and gut microbiota composition. Thus, we can expect that the bile concentration and composition necessary to induce biofilm formation are only reached at certain times or in specific locations in the gut.

Although most studies have been performed with pathogenic bacteria, rather than with symbiotic members of the community, bile was previously shown to induce biofilm formation of at least one representative of all four major bacterial phylum of the gut microbiota: Firmicutes (Clostridioides difficile and Listeria monocytogenes), Bacteroidetes (B. fragilis), Proteobacteria 
(Vibrio cholerae), and Actinobacteria (Bifidobacterium) (15, 17, 26-28), suggesting that bile is an important gut signal for biofilm formation. Consistently, we observed that bile extract induced biofilm formation in almost all tested $B$. thetaiotaomicron strains, as well as in representative strains of most of the common gut Bacteroidales species. Depending on the model considered in each of these studies, the concentration, incubation time, and type of bile component necessary to trigger biofilm formation varied greatly. Bile-dependent biofilm formation also involved different ECM components and different determinants. This suggests that although bile components might be a conserved signal for biofilm formation, the mechanism of biofilm induction differs. Bile could act as a signal triggering biofilm formation to stably colonize the gut. Alternatively, biofilm formation could be a conserved bacterial protection mechanism against the cytotoxic effects of bile, such as lipid membranes and DNA damage and protein denaturation (29). Indeed, bacteria within biofilms are known to be more tolerant to various antimicrobials, including bile acids, than planktonic bacteria, and bile-dependent biofilm formation has been shown to increase tolerance to bile-mediated killing in different species, including $B$. fragilis $(15,17,26)$. Although, the production of a bile salt hydrolase (BSH) (30) and a tripartite multidrug resistance efflux pump, BT2793-2795, confer a high resistance to bile to $B$. thetaiotaomicron, bile-induced biofilm formation in the gut probably constitutes an additional strategy by which gut bacteria protect themselves from bile toxicity (31). Exposure of $B$. thetaiotaomicron to bile also induces various stress responses and increases the production of efflux pumps (25), suggesting the activation of stress tolerance mechanisms that could provide cross-protection against other damaging agents, such as antibiotics $(15,17)$. Interestingly, in Klebsiella pneumoniae, an absence of poly- $N$-acetylglucosamine production significantly reduced bile-dependent biofilm formation in vitro and also impaired its ability to colonize the mouse gut, showing that bile-dependent biofilm formation could impact colonization efficiency (32). Moreover, bile-mediated biofilm formation might be an important aspect of gut microbiota competition and cooperation. Bile is a complex mix of cholesterol, different bile acids, and proteins. Some studies tested purified bile acids, rather than bile extract, and showed that all bacteria did not induce biofilm in response to the same bile acid. For instance, C. difficile reacted to chenodeoxycholate and deoxycholate (17), Bacteroides breve to conjugated bile acids (taurocholate, glycocholate, taurodeoxycholate, and glycodeoxycholate) (33), and Lactobacillus to taurocholate (34). Conversely, taurineconjugated bile acids disperse biofilms of $V$. cholerae or Pseudomonas aeruginosa $(35,36)$. Interestingly, members of the gut microbiota can transform bile acids, with potential consequences on biofilm formation. In particular, several Clostridia can transform primary bile acids, such as cholate, into secondary bile acids, such as deoxycholate, by $7 \alpha$-dehydroxylation, and various bacteria possess a BSH enzyme that can remove the conjugated taurine or glycine residue of specific bile acids (37). These bile modifications by members of the gut microbiota render bile acids less soluble and more cytotoxic, but they could also impact gut microbiota physiology $(38,39)$ and the biofilm formation capacity of other bacteria, and therefore their colonization efficiency. Consistently, Clostridium scindens was shown to increase $C$. difficile biofilm formation in vitro by converting cholate to deoxycholate (17).

Our results indicate that the simultaneous presence of bile, eDNA, and the extracellular DNase BT3563 are required to observe dense, structured B. thetaiotaomicron biofilms. Presence of bile in the growth medium led to important physiological adaptations, as $17 \%$ of B. thetaiotaomicron genes were differentially expressed, but the precise contribution of bile to biofilm formation is still unclear. Although the mechanism of bile-dependent biofilm formation has not been investigated for all bacteria, in some cases, bile was shown to increase the production of exopolysaccharide (32, 40, 41), cyclic-di-GMP (42), and various adhesins such as autotransporters (43) and curli (44). However, very little is known about B. thetaiotaomicron biofilm determinants. Capsular polysaccharide 8 and type $\mathrm{V}$ pili production were previously shown to increase $B$. thetaiotaomicron adhesion capacity, but these genes were not induced in the presence of bile. However, B. thetaiotaomicron mucin-Oglycan-degrading PUL were up-regulated in biofilms grown in chemostats compared to planktonic cultures and in the mucus compared to the lumen $(7,45)$, and they were also induced in the presence of bile, suggesting bile exposure might prime bacteria for adhesion and colonization to the mucus layer in vivo. Bile also increased the release of eDNA, which could modulate biofilm formation. eDNA is a well-known component of the biofilm ECM and numerous studies have shown that addition of DNase to the growth media prevented biofilm formation or dispersed preformed biofilm in both gram-positive and gramnegative bacteria (19). Consistently, addition of DNase I led to a small reduction in the bile-dependent biofilm formation of $B$. thetaiotaomicron, suggesting that eDNA is an important ECM component (Fig. 3B). However, unexpectedly, we show that the extracellular DNase BT3563 is also necessary for biledependent biofilm formation, suggesting that partial degradation of eDNA might be necessary for biofilm formation in the presence of bile. Neither BT3563 transcription nor BT3563 DNase activity was increased by bile, so the link between bile exposure and BT3563 function remains unclear. BT3563 carries a lipoprotein signal commonly found in surface-exposed outer membrane proteins in Bacteroides. Consistently, BT3563 has been detected in the $B$. thetaiotaomicron outer membrane and was enriched in outer-membrane vesicles (OMVs) (46), suggesting BT3563 might be secreted to the supernatant through OMV production. We hypothesize that cell- or OMV-associated BT3563 could degrade eDNA during biofilm formation, maintaining a specific eDNA concentration or organization within the ECM to form structured bile-dependent biofilms. Deletion of BT3563 led to the formation of scattered biofilms in the presence of bile, composed of clumps of cells rather than a dense layer at the bottom of the well. To allow the formation of denser structures, BT3563 might be required to remove eDNA from cell vicinity and prevent electrostatic repulsion between the negatively charged eDNA and bacterial surface or some component of the ECM such as exopolysaccharides (Fig. 6). For instance,

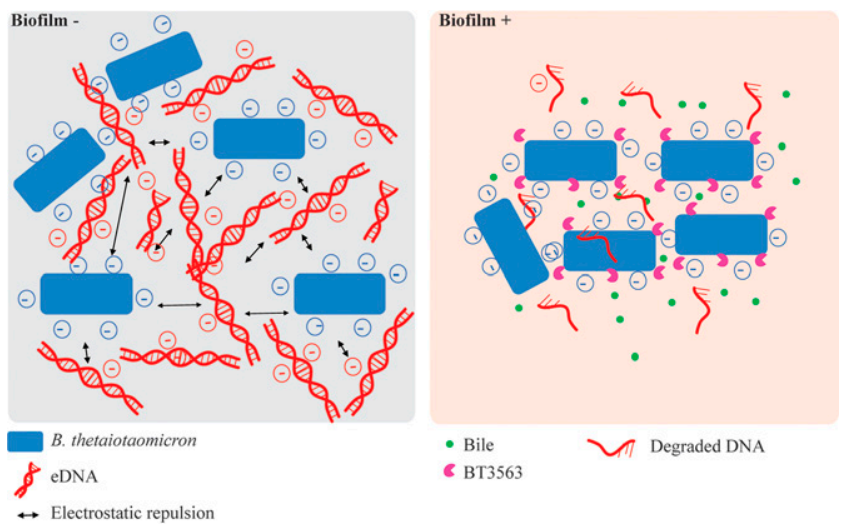

Fig. 6. Model of DNase-mediated biofilm formation. In the absence of BT3563, the electrostatic repulsion between $B$. thetaiotaomicron cells and eDNA prevents close association of cells and biofilm formation. In presence of bile and BT3563, degradation of eDNA surrounding the bacterial cells by BT3563 allows the formation of tight aggregates of cells and biofilm formation. 
the production of positively charged proteins was necessary to prevent electrostatic repulsion between eDNA and Staphylococcus aureus cells in biofilms, and perturbation of these proteins led to an increasingly porous biofilm, reminiscent of our $\triangle B T 3563$ biofilms $(47,48)$. It is also to be noted that a $\triangle B T 3560-3563$ mutant formed less bile-dependent biofilm than a $\triangle B T 3563$ simple mutant, suggesting the TonB-dependent receptor BT3560 and/or the nuclease BT3562 could also contribute to biofilm formation. Moreover, $\triangle B$ T3560-3563 still formed biofilm in the presence of bile, demonstrating that additional factors could be involved.

Our study therefore demonstrates that gut-relevant environmental cues such as bile can strongly stimulate $B$. thetaiotaomicron biofilm formation. This induction relied on the production of an extracellular DNase, BT3563, that is present in all B. thetaiotaomicron isolates, and in many diverse Bacteroidetes. Although our study focused exclusively on in vitro experiments and will therefore need to be confirmed in animal models, our results suggest that, in addition to the well-established structural role of eDNA in the biofilm matrix, partial eDNA degradation might also represent an important aspect of biofilm formation in B. thetaiotaomicron species, and possibly in other Bacteroidales.

\section{Materials and Methods}

Bacterial Strains and Growth Conditions. Bacterial strains used in this study are listed in SI Appendix, Table S3. B. thetaiotaomicron and other Bacteroidales strains were grown in BHIS broth supplemented with erythromycin $15 \mu \mathrm{g} / \mathrm{mL}$ (erm), tetracycline $2.5 \mu \mathrm{g} / \mathrm{mL}$ (tet), gentamycin $200 \mu \mathrm{g} / \mathrm{mL}$ (genta), 5'-fluoro-2'-deoxyruidin $200 \mu \mathrm{g} / \mathrm{mL}$ (FdUR), anhydrotetracycline $(0.1 \mu \mathrm{g} / \mathrm{mL}$ ), D-glucose, D-mannose, D-rhamnose, D-cellobiose, D-maltose $(0.5 \% \mathrm{wt} / \mathrm{vol})$, hemin from bovine $(25 \mathrm{mg} / \mathrm{L}$ and $50 \mathrm{mg} / \mathrm{L})$, porcine mucin extract $(0.1 \%$ and 0 . $5 \% \mathrm{wt} / \mathrm{vol}$ ), bile extract from bovine and ovine (Sigma, B8381) at $0.5 \%$ unless indicated otherwise, DNase I (Thermo Scientific, VF304452) $98 \mathrm{U} / \mathrm{mL}$, or RNase1 (Thermo Scientific, EN0601) $0.06 \mathrm{U} / \mathrm{mL}$ when required. Cultures were incubated at $37^{\circ} \mathrm{C}$ in anaerobic conditions using jars with anaerobic atmosphere generators (GENbag anaero, Biomerieux, No. 45534) or in a C400M Ruskinn anaerobic-microaerophilic station. Escherichia coli S17 $\lambda$ pir was grown in Miller's lysogeny broth (LB) (Corning) supplemented with ampicillin $(100 \mu \mathrm{g} / \mathrm{mL}$ ) when required and incubated at $37^{\circ} \mathrm{C}$ with $180 \mathrm{rpm}$ shaking. Cultures on solid media were done in BHIS with $1.5 \%$ agar, and antibiotics were added when needed. Bacteria were always streaked from glycerol stock on BHIS-agar before being grown in liquid cultures. All media and chemicals were purchased from Sigma-Aldrich unless indicated otherwise.

All experiments and genetic constructions of $B$. thetaiotaomicron were made in VPI-5482 $\Delta t d k$ background (49) unless indicated otherwise, which was developed for a two-step selection procedure of unmarked gene deletion by allelic exchange, as previously described. Therefore, the VPI-5482 $\Delta t d k$ is referred to as wild type or VPI-5482 in this study.

Genome Analysis. Genomic DNA of clinical strains was prepared from overnight cultures using the DNeasy blood and tissue kit (Qiagen). Illumina wholegenome sequencing was performed by the Plateforme de Microbiologie Mutualisée (P2M) of Institut Pasteur and the genomes were assembled using SPAdes v3.13.0 (50) and when necessary were reassembled using Unicycler (51). The obtained genomes were annotated using RASTtk $(52,53)$ on the patricbrc.org database (54) and we searched BT3563 nucleic acid and amino acid sequence in these genomes using the BLASTp tool of patricbrc.org (55, 56). All of these genomes were made available publicly on the patricbrc.org database (see SI Appendix, Table S1 for accession Nos.). Moreover, this wholegenome shotgun project has been deposited at DNA Data Bank of Japan (DDBJ)/European Nucleotide Archive (ENA)/GenBank under the Bioproject accession PRJNA725886 (24). The accession Nos. for each strain are shown in $\mathrm{SI}$ Appendix, Table S1. Sequences of the region surrounding BT3563 homologs were extracted and the gene cluster comparison was made using the clinker and clustermap.js pipeline (57). Synteny analysis was performed using either the web-based tool Synttax (58) searching for homologs of BT3563 using a custom selection of diverse Bacteroidetes or the genome browser tool of the Microscope plateforme (59) looking for B. thetaiotaomicron BT3555-3564 region homology in the seven Bacteroidales strains tested.
A 96-Well Crystal Violet Biofilm Formation Assay. Overnight culture was diluted to $\mathrm{OD}_{600}=0.05$ in $100 \mu \mathrm{L}$ BHIS and inoculated in technical duplicates in polystyrene Greiner round-bottom 96 -well plates. The wells at the border of the plates were filled with $200 \mu \mathrm{L}$ of water to prevent evaporation. Incubation was done at $37^{\circ} \mathrm{C}$ in anaerobic conditions for $48 \mathrm{~h}$. The supernatant was removed by careful pipetting and the biofilms were fixed using $100 \mu \mathrm{L}$ of Bouin's solution (picric acid $0.9 \%$, formaldehyde $9 \%$, and acetic acid $5 \%$; HT10132, Sigma-Aldrich) for $10 \mathrm{~min}$. Then the wells were washed once with water by immersion and flicking, and the biofilm was stained with $125 \mu \mathrm{L} 1 \%$ crystal violet (V5265, Sigma-Aldrich) for $10 \mathrm{~min}$. Crystal violet solution was removed by flicking and biofilms were washed twice with water. Stained biofilms were resuspended in 1:4 acetone:ethanol mix and absorbance at $575 \mathrm{~nm}$ was measured using a TECAN Infinite M200 PRO plate reader.

Targeted Mutagenesis. A list of all the primers used in this study can be found in SI Appendix, Table S4. Deletion mutants in B. thetaiotaomicron VPI 5482 were constructed using the previously described vector for allelic exchange pExchange-tdk (49). Deletion mutants in B. thetaiotaomicron clinical isolates were constructed using the recently described suicide vector pLGB13 for allelic exchange in Bacteroides natural isolates (Addgene 126618) (60). Briefly, 1-kb regions upstream and downstream of the target sequence and the vector (pExchange-tdk or pLGB13) were amplified by PCR using Phusion Flash HighFidelity PCR Master Mix (Thermo Fisher Scientific, F548). All three fragments were ligated using Gibson assembly: the inserts and the plasmids were mixed with Gibson master mix $2 \times(100 \mu \mathrm{L} 5 \times$ ISO buffer, $0.2 \mu \mathrm{L} 10,000 \mathrm{U} / \mathrm{mL}$ T5 exonuclease (NEB No. M0363S), $6.25 \mu \mathrm{L}$ 2,000 U/mL Phusion HF polymerase (NEB No. M0530S), $50 \mu \mathrm{L} 40,000 \mathrm{U} / \mathrm{mL}$ Taq DNA ligase (NEB No. M0208S), $87 \mu \mathrm{L} \mathrm{dH2O}$ for 24 reactions) and incubated at $50^{\circ} \mathrm{C}$ for $35 \mathrm{~min}$. The resulting mix was transformed in $E$. coli S17 $\lambda$ pir that was used to deliver the vector to $B$. thetaiotaomicron by conjugation. Conjugation was carried out by mixing exponentially grown cultures $\left(\mathrm{OD}_{600}=0.6\right)$ of the donor and the recipient strain in a 2 : 1 ratio. The mixture was spotted on BHIS-agar plates and incubated at $37^{\circ} \mathrm{C}$ in aerobic conditions overnight. The mix was then streaked on BHIS agar supplemented with antibiotic-for selection of $B$. thetaiotaomicron transconjugants that had undergone the first recombination event-and gentamicin to ensure exclusion of any $E$. coli growth. Eight of the resulting colonies were grown overnight in BHIS with no antibiotic to allow a second recombination event, and the culture was plated on BHIS-agar plates supplemented with either FdUR (to counterselect pEchange-tdk) or anhydrotetracycline (to counterselect pLGB13) to select for loss of plasmid. The resulting deletion mutants were confirmed by PCR and sequencing.

We used the pNBU2-bla-erm vector (61) or pNBU2-bla-tet (9) vector for complementation, which inserts in the $5^{\prime}$ untranslated region of the tRNA-Ser, in which we previously cloned the constitutive promoter of BT1311 encoding the sigma factor RpoD (8). Target genes were amplified by PCR using Phusion Flash High-Fidelity PCR Master Mix from start codon to stop codon and they were cloned after BT1311 promoter by Gibson assembly. The Gibson mix was transformed in $E$. coli S17 $\lambda$ pir and the resulting $E$. coli was used to transfer the plasmid to $B$. thetaiotaomicron by conjugation (see above).

Random Transposon Mutagenesis. pSAMbt, the previously published tool for random mariner-based transposon mutagenesis in $B$. thetaiotaomicron (62) was conjugated in $B$. thetaiotaomicron as described above. After streaking on BHIS-erm-genta agar plates, isolated colonies were resuspended in $100 \mu \mathrm{L}$ BHIS in 96-well plates, grown overnight, and tested for biofilm formation as described above. The selected clones were then streaked on a fresh BHIS-ermgenta agar plate and three isolated colonies were tested for biofilm formation to ensure no mix of transposon mutants had occurred during preparation of the library. The genomic DNA of the validated clones was extracted using the DNeasy blood and tissue kit (Qiagen) and sent for whole-genome sequencing at the Mutualized Platform for Microbiology of Institut Pasteur.

Growth Curve. A total of $2.5 \mu \mathrm{L}$ of overnight cultures was added to $200 \mu \mathrm{L}$ BHIS that had previously been incubated in anaerobic condition overnight to remove dissolved oxygen, in Greiner flat-bottom 96-well plates. A plastic adhesive film (adhesive sealing sheet; Thermo Scientific, AB0558) was added on top of the plate inside the anaerobic station, and the plates were then incubated in a TECAN Infinite M200 Pro spectrophotometer for $24 \mathrm{~h}$ at $37^{\circ} \mathrm{C} . \mathrm{OD}_{600}$ was measured every $30 \mathrm{~min}$, after a 900 -s orbital shaking of $2 \mathrm{~mm}$ amplitude.

Viable Cells Count. Overnight cultures were diluted to $\mathrm{OD}_{600}=0.5$ in a final volume of $1 \mathrm{~mL}$ of $\mathrm{NaCl} 0.85 \%$. Aliquots were centrifugated at $6,000 \times g$ $(7,000 \mathrm{rpm})$ for $7 \mathrm{~min}$ and the supernatant was discarded. After washing twice with $\mathrm{NaCl} 0.85 \%, 2 \mu \mathrm{L}$ of propidium iodide was added and the samples were incubated in anaerobic condition and protected from light at $37^{\circ} \mathrm{C}$, for 
$30 \mathrm{~min}$. The number of viable bacteria was then quantified using a MACSQuant VYB flow cytometer and MACSQuantify Software V2.11.

Extracellular Matrix Extraction and Quantification. ECMs were extracted based on previously described methods (63). A total of $2 \mathrm{~mL}$ of 48-h biofilm cells grown in the presence of bile were harvested and centrifugated at $5,000 \times g$ for $10 \mathrm{~min}$. The pellet was washed twice with $\mathrm{NaCl} 0.85 \%$ and weighed, then resuspended in an extraction buffer (Tris. $\mathrm{HCl} \mathrm{pH} 8.0 ; 1.5 \mathrm{M}$ $\mathrm{NaCl}$ ) at a 1:10 mass-volume ratio and incubated at $25^{\circ} \mathrm{C}$ for $30 \mathrm{~min}$ with agitation. Then, cells were removed by centrifugation at $15,000 \times g$ and $25^{\circ} \mathrm{C}$ for $10 \mathrm{~min}$ and the supernatant containing the extracted ECMs was stored at $-20^{\circ} \mathrm{C}$ until use. The amount of DNA, and proteins in the ECM were measured using a Qubit 3.0 fluorometer (Thermo Fisher Scientific) according to the manufacturer's instructions.

Nuclease Activity of the Supernatant. Overnight or 48-h cultures were centrifugated for $6.5 \mathrm{~min}$ at $6,000 \times \mathrm{g}$. When applicable, we measured eDNA concentration using the QuBit HS double-stranded DNA kit (Invitrogen). A total of $50 \mu \mathrm{L}$ of supernatant was mixed with $B$. thetaiotaomicron VPI-5482 genomic DNA. $10 \mu \mathrm{L}$ was used to run on a $1 \%$ agarose gel and colored with ethidium bromide. The remaining $40 \mu \mathrm{L}$ was incubated at $37^{\circ} \mathrm{C}$ overnight for approximatively $18 \mathrm{~h}$, and then $10 \mu \mathrm{L}$ was used to run a $1 \%$ agarose gel and colored with ethidium bromide. DNase I was used as a positive control of DNase activity.

CLSM. For confocal laser scanning microscopy, biofilms were grown in 96-well plates ( $\mu$ clear, Greiner Bio-One). A total of $100 \mu \mathrm{L}$ of BHIS, supplemented with $0.5 \%$ bile extract, DNase I (Thermo Scientific, VF304452) $98 \mathrm{U} / \mathrm{mL}$, or RNase1 (Thermo Scientific, EN0601) $0.06 \mathrm{U} / \mathrm{mL}$ when required, was added to each well and the plates were incubated at $37^{\circ} \mathrm{C}$, in static condition $48 \mathrm{~h}$ under anaerobic conditions. The unwashed biofilms were then directly stained in red with $20 \mu \mathrm{M}$ of SYTO61 (Life Technologies; cell permeant nucleic acid dye to contrast all the bacteria) and in green with $0.4 \mu \mathrm{M}$ of TOTO-1 (Thermo Scientific; cell impermeant DNA dye to contrast eDNA). After $15 \mathrm{~min}$ of incubation, $Z$ stacks of horizontal plane images were acquired in 1- $\mu$ m steps using CLSM (Leica TCS SP8, INRAE MIMA2 microscopy platform) with a water $63 \times$ immersion lens (numerical aperture $[N A]=1.2$ ). Two stacks of images were acquired randomly on three independent samples at $800 \mathrm{~Hz}$. Fluorophores were excited and

1. P. B. Eckburg et al., Diversity of the human intestinal microbial flora. Science $\mathbf{3 0 8}$ 1635-1638 (2005)

2. H. M. Wexler, Bacteroides: The good, the bad, and the nitty-gritty. Clin. Microbiol. Rev. 20, 593-621 (2007)

3. E. C. Martens, H. C. Chiang, J. I. Gordon, Mucosal glycan foraging enhances fitness and transmission of a saccharolytic human gut bacterial symbiont. Cell Host Microbe 4, 447-457 (2008)

4. S. Macfarlane, G. T. Macfarlane, Composition and metabolic activities of bacterial biofilms colonizing food residues in the human gut. Appl. Environ. Microbiol. 72 6204-6211 (2006).

5. J. L. Sonnenburg et al., Glycan foraging in vivo by an intestine-adapted bacterial symbiont. Science 307, 1955-1959 (2005)

6. J. L. Mark Welch, Y. Hasegawa, N. P. McNulty, J. I. Gordon, G. G. Borisy, Spatial organization of a model 15-member human gut microbiota established in gnotobiotic mice. Proc. Natl. Acad. Sci. U.S.A. 114, E9105-E9114 (2017).

7. M. A. TerAvest et al., Regulated expression of polysaccharide utilization and capsular biosynthesis loci in biofilm and planktonic Bacteroides thetaiotaomicron during growth in chemostats. Biotechnol. Bioeng. 111, 165-173 (2014).

8. J. Mihajlovic et al., A putative type v pilus contributes to Bacteroides thetaiotaomicron biofilm formation capacity. J. Bacteriol. 201, e00650-18 (2019).

9. N. Béchon et al., Capsular polysaccharide cross-regulation modulates Bacteroides thetaiotaomicron biofilm formation. MBio 11, e00729-20 (2020).

10. J. M. Grondin, K. Tamura, G. Djean, D. W. Abbott, H. Brumer, Polysaccharide utilization loci: Fueling microbial communities. J. Bacteriol. 199, e00860-16 (2017)

11. N. Bollinger, D. J. Hassett, B. H. Iglewski, J. W. Costerton, T. R. McDermott, Gene expression in Pseudomonas aeruginosa: Evidence of iron override effects on quorum sensing and biofilm-specific gene regulation. J. Bacteriol. 183, 1990-1996 (2001).

12. R. R. Bollinger et al., Secretory IgA and mucin-mediated biofilm formation by environmental strains of Escherichia coli: Role of type 1 pili. Mol. Immunol. 43, 378-387 (2006)

13. S. J. Ahn, R. A. Burne, Effects of oxygen on biofilm formation and the AtlA autolysin of Streptococcus mutans. J. Bacteriol. 189, 6293-6302 (2007).

14. L. R. Hoffman et al., Aminoglycoside antibiotics induce bacterial biofilm formation Nature 436, 1171-1175 (2005).

15. L. Pumbwe et al., Bile salts enhance bacterial co-aggregation, bacterial-intestinal epithelial cell adhesion, biofilm formation and antimicrobial resistance of Bacteroides fragilis. Microb. Pathog. 43, 78-87 (2007). emissions were captured as prescribed by the manufacturer. Simulated 3D fluorescence projections were generated using IMARIS 9.3 software (Bitplane).

RNA-Seq Analysis. Overnight cultures were mixed with RNAprotect (Qiagen) to prevent RNA degradation, and the pellet was kept at $-80^{\circ} \mathrm{C}$. Total RNA was extracted using MP Biomedicals FastRNA Pro Blue kit by the provider's manual and treated with an Ambion Turbo DNA-free kit to remove possible DNA contamination. Total RNA from four independent replicates was checked on RNA6000 Nano chips on a Bioanalyzer (Agilent) for its quality and integrity. Ribosomal RNA depletion was performed using the Bacteria RiboZero kit (Illumina). From rRNA-depleted RNA, directional libraries were prepared using the TruSeq Stranded mRNA Sample preparation kit following the manufacturer's instructions (Illumina). Libraries were checked for quality on Bioanalyzer DNA 1000 chips (Agilent). Quantification was performed with the fluorescent-based quantitation Qubit dsDNA HS Assay kit (Thermo Fisher Scientific). Sequencing was performed as a single read run for 65 -bp sequences on a HiSEq 2500 Illumina sequencer (65 cycles). The multiplexing level was 16 samples per lane. Bioinformatics analysis was performed using the RNA-seq pipeline from Sequana.

Data Availability. All study data are included in the article and/or supporting information. The genomes of strains sequenced in this article are available at https://www.ncbi.nlm.nih.gov/bioproject/?term=PRJNA725886 (24).

ACKNOWLEDGMENTS. We thank Rebecca Stevick, Christophe Beloin, Susan Joyce, and David Clarke for critical reading of the manuscript. We are grateful to Andy Goodman, Justin Sonnenburg, and Laurie Comstock for providing the genetic tools used in this study. This work was supported by an Institut Pasteur grant and by the French government's Investissement d'Avenir Program, Laboratoire d'Excellence "Integrative Biology of Emerging Infectious Diseases" (Grant ANR-10-LABX-62-IBEID) and the Fondation pour la Recherche Médicale (Grant DEQ20180339185). N.B. was supported by a Ministère Français de I'Education Nationale, de l'Enseignement Supérieur et de la Recherche fellowship. J.M. was supported by the Pasteur Paris University International Doctoral Program and the Fondation pour la Recherche Médicale (Grant FDT20160435523). The RNA-seq experiments were performed by the Biomics Platform, C2RT, Institut Pasteur, Paris, France, supported by France Génomique (Grant ANR10-INBS-09-09) and Infrastructures en Biologie Santé et Agronomie (IBISA).

16. J. F. Sicard, G. L. Bihan, P. Vogeleer, M. Jacques, J. Harel, Interactions of intestinal bac teria with components of the intestinal mucus. Front Cell Infect. Microbiol. 7, 387 (2017).

17. T. Dubois et al., A microbiota-generated bile salt induces biofilm formation in Clostridium difficile. npj Biofilms Microbiomes 5, 14 (2019).

18. J. O. Silva et al., In vitro effect of antibiotics on biofilm formation by Bacteroides fragilis group strains isolated from intestinal microbiota of dogs and their antimicrobia susceptibility. Anaerobe 28, 24-28 (2014).

19. M. Okshevsky, R. L. Meyer, The role of extracellular DNA in the establishment, maintenance and perpetuation of bacterial biofilms. Crit. Rev. Microbiol. 41, 341-352 (2015).

20. H. Liu et al., Functional genetics of human gut commensal Bacteroides thetaiotaomicron reveals metabolic requirements for growth across environments. Cell Reports 34, 108789 (2021).

21. J. Xu et al., A genomic view of the human-Bacteroides thetaiotaomicron symbiosis. Science 299, 2074-2076 (2003).

22. M. J. Coyne, L. E. Comstock, Niche-specific features of the intestinal bacteroidales. J. Bacteriol. 190, 736-742 (2008)

23. Q. Xu et al., A distinct type of pilus from the human microbiome. Cell 165, 690-703 (2016).

24. N. Béchon, et al., DNA Data Bank of Japan (DDBJ)/European Nucleotide Archive (ENA)/GenBank. https://www.ncbi.nlm.nih.gov/bioproject/?term=PRJNA725886. Deposited 28 April 2021

25. S. M. Kristoffersen et al., Low concentrations of bile salts induce stress responses and reduce motility in Bacillus cereus ATCC 14570. J. Bacteriol. 189, 5302-5313 (2007).

26. D. T. Hung, J. Zhu, D. Sturtevant, J. J. Mekalanos, Bile acids stimulate biofilm formation in Vibrio cholerae. Mol. Microbiol. 59, 193-201 (2006).

27. M. Begley, C. Kerr, C. Hill, Exposure to bile influences biofilm formation by Listeria monocytogenes. Gut Pathog. 1, 11 (2009)

28. P. Ambalam, K. K. Kondepudi, I. Nilsson, T. Wadström, A. Ljungh, Bile enhances cell surface hydrophobicity and biofilm formation of bifidobacteria. Appl. Biochem. Biotechnol. 172, 1970-1981 (2014).

29. V. Urdaneta, J. Casadesús, Interactions between bacteria and bile salts in the gastrointestinal and hepatobiliary tracts. Front. Med. (Lausanne) 4, 163 (2017).

30. L. Yao et al., A selective gut bacterial bile salt hydrolase alters host metabolism. eLife 7, e37182 (2018)

31. H. C. Flemming et al., Biofilms: An emergent form of bacterial life. Nat. Rev. Microbiol. 14, 563-575 (2016) 
32. K. M. Chen et al., The role of pgaC in Klebsiella pneumoniae virulence and biofilm formation. Microb. Pathog. 77, 89-99 (2014).

33. S. M. Kelly et al., Bifidobacterial biofilm formation is a multifactorial adaptive phenomenon in response to bile exposure. Sci. Rep. 10, 11598 (2020).

34. P. Ambalam, K. K. Kondepudi, I. Nilsson, T. Wadström, Å. Ljungh, Bile stimulates cell surface hydrophobicity, Congo red binding and biofilm formation of Lactobacillus strains. FEMS Microbiol. Lett. 333, 10-19 (2012).

35. A. J. Hay, J. Zhu, Host intestinal signal-promoted biofilm dispersal induces Vibrio cholerae colonization. Infect. Immun. 83, 317-323 (2015).

36. L. M. Sanchez et al., Biofilm formation and detachment in gram-negative pathogens is modulated by select bile acids. PLoS One 11, e0149603 (2016).

37. N. Molinero, L. Ruiz, B. Sánchez, A. Margolles, S. Delgado, Intestinal bacteria interplay with bile and cholesterol metabolism: Implications on host physiology. Front. Physiol. 10, 185 (2019).

38. D. Zhu, J. A. Sorg, X. Sun, Clostridioides difficile biology: Sporulation, germination, and corresponding therapies for C. difficile infection. Front Cell Infect. Microbiol. 8 , 29 (2018).

39. S. Cheng, L. Zhu, H. S. Faden, Interactions of bile acids and the gut microbiota: Learning from the differences in Clostridium difficile infection between children and adults. Physiol. Genomics 51, 218-223 (2019).

40. R. W. Crawford, D. L. Gibson, W. W. Kay, J. S. Gunn, Identification of a bile-induced exopolysaccharide required for Salmonella biofilm formation on gallstone surfaces. Infect. Immun. 76, 5341-5349 (2008).

41. K. P. Nickerson et al., Analysis of Shigella flexneri resistance, biofilm formation, and transcriptional profile in response to bile salts. Infect. Immun. 85, e01067-16 (2017).

42. B. J. Koestler, C. M. Waters, Bile acids and bicarbonate inversely regulate intracellular cyclic di-GMP in Vibrio cholerae. Infect. Immun. 82, 3002-3014 (2014).

43. V. K. Köseoğlu, C. P. Hall, E. M. Rodríguez-López, H. Agaisse, The autotransporter IcsA promotes shigella flexneri biofilm formation in the presence of bile salts. Infect. Immun. 87, e00861-18 (2019).

44. J. F. González et al., Human bile-mediated regulation of Salmonella curli fimbriae. J. Bacteriol. 201, e00055-19 (2019).

45. H. Li et al., The outer mucus layer hosts a distinct intestinal microbial niche. Nat. Commun. 6, 8292 (2015)

46. E. Valguarnera, N. E. Scott, P. Azimzadeh, M. F. Feldman, Surface exposure and packing of lipoproteins into outer membrane vesicles are coupled processes in Bacteroides. MSphere 3, e00559-18 (2018).

47. V. Dengler, L. Foulston, A. S. DeFrancesco, R. Losick, An electrostatic net model for the role of extracellular DNA in biofilm formation by Staphylococcus aureus. J. Bacteriol. 197, 3779-3787 (2015).
48. J. S. Kavanaugh et al., Identification of extracellular DNA-binding proteins in the biofilm matrix. MBio 10, e01137-19 (2019).

49. N. M. Koropatkin, E. C. Martens, J. I. Gordon, T. J. Smith, Starch catabolism by a prom inent human gut symbiont is directed by the recognition of amylose helices. Struc ture 16, 1105-1115 (2008).

50. A. Bankevich et al., SPAdes: A new genome assembly algorithm and its applications to single-cell sequencing. J. Comput. Biol. 19, 455-477 (2012)

51. R. R. Wick, L. M. Judd, C. L. Gorrie, K. E. Holt, Unicycler: Resolving bacterial genome assemblies from short and long sequencing reads. PLOS Comput. Biol. 13, e1005595 (2017).

52. T. Brettin et al., RASTtk: A modular and extensible implementation of the RAST algorithm for building custom annotation pipelines and annotating batches of genomes. Sci. Rep. 5, 8365 (2015)

53. A. R. Wattam et al., Improvements to PATRIC, the all-bacterial bioinformatics database and analysis resource center. Nucleic Acids Res. 45 (D1), D535-D542 (2017).

54. J. J. Davis et al., The PATRIC Bioinformatics Resource Center: Expanding data and analysis capabilities. Nucleic Acids Res. 48 (D1), D606-D612 (2020)

55. G. M. Boratyn et al., BLAST: A more efficient report with usability improvements. Nucleic Acids Res. 41, W29-W33 (2013).

56. N. A. O'Leary et al., Reference sequence (RefSeq) database at NCBI: Current status, taxonomic expansion, and functional annotation. Nucleic Acids Res. 44 (D1) D733-D745 (2016).

57. C. L. M. Gilchrist, Y.-H. Chooi, Clinker \& clustermap.js: Automatic generation of gene cluster comparison figures. Bioinformatics 37, 2473-2475 (2021). 10.1093/ bioinformatics/btab007

58. J. Oberto, SyntTax: A web server linking synteny to prokaryotic taxonomy. BMC Bioinformatics 14, 4 (2013).

59. D. Vallenet et al., Microscope: An integrated platform for the annotation and exploration of microbial gene functions through genomic, pangenomic and metabolic comparative analysis. Nucleic Acids Res. 48 (D1), D579-D589 (2020).

60. L. García-Bayona, L. E. Comstock, Streamlined genetic manipulation of diverse bac teroides and parabacteroides isolates from the human gut microbiota. MBio 10 e01762-19 (2019)

61. J. Wang, N. B. Shoemaker, G. R. Wang, A. A. Salyers, Characterization of a Bacteroides mobilizable transposon, NBU2, which carries a functional lincomycin resistance gene. J. Bacteriol. 182, 3559-3571 (2000).

62. A. L. Goodman et al., Identifying genetic determinants needed to establish a human gut symbiont in its habitat. Cell Host Microbe 6, 279-289 (2009)

63. A. Chiba, S. Sugimoto, F. Sato, S. Hori, Y. Mizunoe, A refined technique for extraction of extracellular matrices from bacterial biofilms and its applicability. Microb. Biotechnol. 8, 392-403 (2015). 